\title{
Evaluating Kinship Care Alternatives: A Comparison of a Private Initiative to Traditional State Services
}

\author{
Rodney A. Ellis \\ Gary D. Ellis \\ Rochelle Galey
}

\begin{abstract}
Multiplestressors on the child welfaresystem haveforced innovativesolutions to the overburdened foster care program. A promising alternative is kinship care, in which children are placed with biological relatives. Proponents cite the opportunity to placethechild in familiar surroundings, the natural access to additional family resources, and the degreeto which it is sensitiveto the norms and values of non-dominant cultural groups. Various models of kinship care have been implemented in several jurisdictions, yet littleor no research has been doneto determine which alternatives are the most effective. This paper addresses that deficit. It reports the results of a study that comparesstability of placement outcomes between a program operated by a private, not-for-profit organization $(n=60)$ and a moretraditional program ( $n=79)$ operated by a state child welfare agency. Results support the use of the private alternative over themore traditional state-operated program.
\end{abstract}

Keywords: Kinship care, child welfare, foster care, at-risk children, children's services, placement

T he child welfare system in the United States has recently experienced a myriad of stressors. The number of children in care has skyrocketed, as has the number of children with special needs (Courtney, 1996; United States Department of Health and Human Services, 1996). Experts cite large numbers of children with special education or mental health issues (Chernoff, Combs-Orme, Risley-Curtiss \& Heisler, 1994), an influx of substance-exposed infants (Ross, 1995), and children with serious health problems (Bilaver, Jaudes, Koepke \& Goerge, 1999). Increasing numbers of minorities have entered the system (Scannapieco \& Jackson, 1996), raising questions about the appropriateness of traditional programs. The system, never rich in resources, has been hard-pressed to respond.

Kinship care, the placement of at-risk children with biological relatives, has been lauded as a partial, yet effective response (National Adoption Information Clearinghouse [NAIC], 1997). Relatives provide a familiar, comfortable environ-

Rodney A. Ellis, Ph.D. is Assistant Professor, The University of Tennessee College of Social Work. Gary D. Ellis, Ph.D. is Professor and Chair, University of Utah Department of Parks, Recreation, and Tourism. Rochelle Galey, M.S.S.W., is an alcohol and substance abuse counselor in Colorado Springs, Colorado.

Copyright 2002 Advances in Social Work Vol. 3 No. 1 (Spring 2002) 33-45.

Indiana University School of Social Work. 
ment, lessening the trauma children experience from forced relocation (Ingram, 1996). Family members may know of others who can help with transportation, supervision, or financial needs. Placement with relatives may help to address cultural issues, allowing the child to remain in a culturally similar environment (Child Welfare League of America, 1994). Perhaps most important to the overburdened system, a diligent search for relatives can help to generate placement alternatives, directing children away from the traditional system.

Kinship care is not a new concept. Children in need of protection have been removed from their homes and placed with relatives throughout the history of child welfare. What is new, however, are the kinds of resources directed to caregivers (Gleeson, 1999; Ingram, 1996). Some programs are exploring alternative management processes, such as family mediation (Scannapieco, 1999; Wilhelmus, 1998). Others use family meetings for decision-making and support long-term care as an alternative to adoption. Many offer a variety of new resources, such as financial assistance, caregiver support groups, and parenting courses (Scannapieco, 1999).

Despite the enthusiasm with which new approaches to kinship care have been greeted, few researchers have compared the effectiveness of various program alternatives. This paper reports the results of different outcomes of two kinship programs. Both operated in Davidson County, Tennessee (Nashville). One program, herein identified as "public," served children placed in the temporary custody of the Tennessee Department of Children's Services, which then encouraged relatives to provide care and seek permanent custody of the children. This program offered a more traditional style of supervision, including case management, emergency financial support, clothing, and transportation. The second program ("private") was operated by a partnership among three private agencies, the Court Appointed Special Advocate program (CASA), the Vanderbilt Legal Clinic, and Family and Children's Services. The privately sponsored Relative Caregiver Program offered the same basic services as did the public program, but also included a broader range of services, including linkage to CASA volunteers (provided by CASA), weekly support group meetings, therapeutic activity groups, family and individual counseling, educational training (provided by Family and Children's Services), and legal representation (provided by the Vanderbilt Legal Clinic).

\section{LITERATURE REVEW}

The kinship literature features a variety of publication types, including: 1) studies of children, caregivers, and biological parents, 2) program descriptions, and 3) outcome evaluations. Because of their value in conceptualizing this study, the literature in each of these categories is reviewed here. In addition, potential outcome variables are identified.

\section{Descriptive Studies}

Several studies have examined the characteristics of children in relative care, their caregivers, and the parents whose children are placed with kin. Children's studies have focused on demographics, medical conditions, and behavioral or psychoso- 
cial problems (Berrick, Barth, \& Needell, 1994; Dubowitz, Tepper, Feigelman, Sawyer \& Davidson, 1990; Gabel, 1992; I glehart, 1994; Task Force on Permanency Planning for Foster Children [TFPPFC], 1990). Caregiver studies have reviewed demographics, commitment to caregiving, and willingness to comply with traditional permanency alternatives (Berrick, et al., 1994; Dubowitz, et al., 1990; Gabel, 1992; I glehart, 1994; TFPPFC, 1990). Research regarding biological parents has been primarily limited to demographics (Berrick, etal., 1994; Dubowitz, etal., 1990; Gabel, 1992; I glehart, 1994; TFPPFC, 1990; Thornton, 1991; Wulczyn \& Goerge, 1990).

The mean age of children in kinship care is seven to eight years (Berrick, et al., 1994; Dubowitz, et al., 1990; Iglehart, 1994; TFPPFC, 1990). They are predominantly African-American (Berrick, et al., 1994; Iglehart, 1994). Distribution by gender appears to be equal (Berrick, et al., 1994; Dubowitz, et al., 1990). Most of the children are in custody due to neglect or parental substance abuse (Berrick, et al., 1994; Gabel, 1992; TFPPFC, 1990). Often, the substance abuse has resulted in prenatal exposure for the child (Berrick, et al., 1994; Gabel, 1992; TFPPFC, 1990; Thornton, 1991).

Regarding psychosocial issues, conclusions have been inconsistent. In the area of physical health, Berrick, et al. (1994) found that most children were free from significant physical problems. Dubowitz, et al. (1992), however, found that only $10 \%$ of the children they studied were free from medical problems. Scannapieco, Hegar and McAlpine (1997) suggest that results may vary because of different sources of assessment data.

Several researchers have identified behavioral problems. Dubowitz, et al. (1990) found that 35 percent of the children in kinship carescored in the clinical range on the Child Behavior Checklist. Berrick, et al . (1994) concluded that children in relative care scored more than one standard deviation above the norm on the Behavior Problem Index. However, both Berrick, et al. (1994) and Iglehart (1994) found fewer problems among children in kinship care than among those in foster care. In school, 60 percent of the children in kinship care behaved satisfactorily (Berrick, et al., 1994; Dubowitz, et al., 1990; Iglehart, 1994), but between 36 and 50 percent performed below grade level in academic performance (Dubowitz, et al., 1990; I glehart, 1994).

Caregivers have been primarily African-American, about 50 years old, and female (Berrick, et al., 1994; Dubowitz, et al., 1990; Gabel, 1992; TFPPFC, 1990). Most have been maternal grandmothers (over 50\%) and aunts (up to 33\%) (Dubowitz, et al., 1990; Gabel, 1992; TFPPFC, 1990; Thornton, 1991). Most have been single, and high school graduates (Berrick, et al., 1994; Dubowitz, et al., 1990; Gabel, 1992). Many were employed, yet lived at or below the poverty line (Berrick, et al., 1994; Dubowitz, et al., 1990). Regarding willingness to comply with child welfare goals, many caregivers were willing to provide long-term care (Berrick, et al., 1994; Dubowitz, et al., 1990; Thornton, 1991) but were reluctant to adopt or assume legal guardianship (Berrick, et al., 1994; I glehart, 1994; Thornton, 1991).

Biological parents were predominantly African-American, with a mean age of 27 years. Their primary income was from income maintenance programs. Many could not be located when their children were taken into custody (Gabel, 1992; 
TFPPFC, 1990). Although different reasons were cited for removal of children, the primary reason was neglect, often compounded by parental substance abuse (Berrick, et al., 1994; Gabel, 1992; Iglehart, 1994; Thornton, 1991; Wulczyn \& Goerge, 1990).

\section{Program Descriptions}

The literature regarding kinship program descriptions has been summarized by Scannapieco (1999). She identifies two categories of programs, one distinguished by funding source and a second defined by continuum of service. Similar typologies will be needed to facilitate outcome comparisons of models. Review of the current literature suggests at least four programmatic dimensions. They include: 1) formal vs. informal programs, 2) types and ranges of services, 3) identity of service providers, and 4) program goals and philosophies.

Formal Versus Informal Programs

Placement with relatives has long been an alternative. In some cases, placements have been arranged without court involvement or state supervision. These placements have been termed informal (NAIC, 1997). In other cases, children have been placed by court order, and ongoing state supervision has been mandated (NAIC, 1977). These arrangements are considered formal. Generally, when professionals refer to kinship care programs, they mean formal programs where judicial order and state responsibility are present.

Types and Range of Services

Programs offer a range of services. They may include a monthly stipend, case management, emergency financial relief, support groups, counseling services, parenting classes, educational services, and others (Wilson, 1999). For example, Scannapieco, et al. (1997) identified the services offered to Baltimore kinship homes as medical and mental health, education, transportation, in-home aid, housing, crisis intervention, and parent education. Service delivery models ranged from directive case management to mediation (Wilhelmus, 1998).

The variability of service availability among programs raises the question of the contribution of each to outcome. In multi-faceted interventions, it is critical to know which components are effective and which are ineffective. For example, stipends may enable families with limited resources to accept a child when it might not otherwise be possible. Similarly, programs that include support groups may help caregivers deal with stressors that might otherwise disrupt the placement.

\section{Identity of ServiceProviders}

Another important dimension is the identity of the service provider. In some programs, the primary service-delivery organization is a government agency. In others, it is private (Wilson, 1999). The primary agency may directly offer a broad range of services or may broker most services among a network of agencies. Similarly, some may provide the majority of services through a case manager, while others may serve primarily as a source of referrals. 


\section{Program Philosophies and Goals}

Program philosophies and goals differ among programs. Philosophical bases include family preservation, diversion from out-of-home care, or out-of-home care (Scannapieco, 1999; Scannapieco \& Hegar, 1996). Goals may include reunification with parents, long-term placement with relatives, overall placement stability, preservation of family ties, adoption, independent living, reduction of the trauma generated by removal, and ongoing contact with the child's natural culture (Gleeson \& Craig, 1994; Hegar, 1999; Ingram, 1996; Scannapieco, 1999).

\section{OutcomeEvaluations}

Few kinship outcome evaluations have been published. Most have defined success in terms of duration and stability of placement (Scannapieco, et al., 1997). These measures are closely related to program goals such as placement stability, preservation of family relationships, adoption, trauma reduction, and cultural sensitivity. Previous research has compared placement stability in kinship homes to that in traditional foster homes. Kinship placements have been more stable than traditional placements (Berrick, et al., 1994; Dubowitz, et al., 1990; Gabel, 1992; TFPPFC, 1990; Wulczyn \& Goerge, 1990). The current researchers were unable to locate any studies reporting the results of outcomes between kinship care models.

Kinship care is likely to be a permanent child welfare strategy (Scannapieco, 1999). This makes comparisons between kinship and traditional programs far less relevant. In fact, Dubowitz (1994) observed that, "rather than asking whether kinship care is good or bad, the important policy-relevant question is what factors influence the success or failure of kinship placements" (p. 562). This suggests that future research should examine effectiveness among program alternatives as well as the elements of successful programs.

A single outcome study focused on quality of care. Berrick (1997) found that children in kinship settings were less safe than children in traditional settings (based on such factors as the presence of a first-aid kit, caretaker knowledge of CPR, and the physical environment of the home and community). However, kinship homes ranked higher in terms of both presence of and quality of a relationship with the mother.

\section{Identification of OutcomeVariables}

The literature suggests that a broad selection of outcome variables is relevant. Dimensions from which variables might be selected include: 1) characteristics of children (such as psychosocial functioning, quality of care [Berrick, 1997], medical and behavioral conditions [Bilaver, et al., 1999]); 2) conditions of the family (relationships [Berrick, 1997; Ingram, 1996] , contact with biological parent [Berrick, 1997]); and 3) conditions of the placement (safety [Berrick, 1997], stability of placement [Berrick, 1997; Henry, 1999; Gleeson \& Craig, 1994]). Ideally, evaluations should include a combination of these variables, but the nature of the data and sample size often inhibit choice. Stability of placement is itself a valuable measure, and may serve as a proxy for others, such as psychosocial functioning, quality of care, and family relationships. Evidence of its suitability as a proxy can be found in 
studies that have shown placement disruption can have a negative effect on the psychological, emotional, and intellectual development of children (Ainsworth, Blehar, Waters \& Wall, 1978; Fahlberg, 1979; Goldstein, Freud \& Solnit, 1973; Verschueren, Marcoen \& Schoefs, 1996).

\section{METHODOLOGY}

\section{Sample}

The data were abstracted from the private program files and Davidson County Juvenile Court records. Data collectors were trained in abstraction techniques and closely supervised to minimize the probability of problems with inter-rater reliability. Training techniques included: 1) a thorough review of the document containing the original data, 2) trial efforts in data collection using fabricated data, and 3) a discussion of how discrepancies in the original data should be handled. Supervisory techniques involved the presence of the same supervisor during each data collection period. The supervisor answered questions and reviewed random data collection forms for clarity and accuracy. Some inconsistencies may have existed in the way in which data were originally collected by workers from the private program or the juvenile court.

The sample consisted of 148 children between infancy and age 18. Nine children who had received services from both the private and the public programs were excluded from the analysis. Each of the participants represented a distinct kinship placement, that is, neither group's total included settings in which siblings were placed together in the home of a relative. Table 1 reports distribution by age for the 139 participants. Seventy-five (54\%) were female and 64 (46\%) were male (see Table 2). They were predominantly African-American ( $n=113,81.3 \%)$, and included 23 Caucasians (16.5\%), one Native American/Alaska Native (.7\%), one who selfidentified as Multi-racial (.7\%), and one who self-identified as Other (.7\%). Distribution by race is reported in Table 3. All were residents of the Metropolitan Davidson County (Nashville) area. The private group $(n=79)$ included all children who participated in the program between August, 1997 and February, 1999. The public group $(n=60)$ consisted of a randomly selected sample of children who received services from that program during the same period. Outcome data were collected through May 2000.

\section{Variable Selection, Research Design, and Hypothesis}

Since randomization was not possible, the researchers selected three variables to determine group equivalency. Age, sex, and race were used for comparison. Age was continuous, including children from infancy to 18 years. Sex was, of course, dichotomous. Race was categorical, the categories having been determined by program documents. Categories included Asian, Native American or Alaska Native, Black, Hispanic, Hawaiian or Pacific Islander, Multiracial, Caucasian, and Other.

Positive outcome was defined as stability of placement. Stability of placement was defined as remaining consistently within the same home without permanent removal to an alternative setting. Disruption for any reason other than return to a biological parent was regarded as unsuccessful. The dependent variable was, 


\begin{tabular}{|ccccccc|}
\hline Table 1: & Distribution of the Sampleby Age & \multicolumn{5}{c|}{ Total } \\
\hline Age & \multicolumn{2}{c}{ PRIVATE } & \multicolumn{2}{c}{ PUBLIC } & \% & $\mathbf{n}$ \\
& $\%$ & $\mathbf{n}$ & $\%$ & $\mathbf{n}$ & 2.9 & 4 \\
2 & 3.8 & 3 & 1.7 & 1 & 6.5 & 9 \\
3 & 6.3 & 5 & 6.7 & 4 & 5.0 & 7 \\
4 & 6.3 & 5 & 3.3 & 2 & 3.6 & 5 \\
5 & 5.1 & 4 & 1.7 & 1 & 5.8 & 8 \\
6 & 1.3 & 1 & 11.7 & 7 & 5.0 & 7 \\
7 & 5.1 & 4 & 5.0 & 3 & 7.2 & 10 \\
8 & 8.9 & 7 & 5.0 & 3 & 6.5 & 9 \\
9 & 6.3 & 5 & 6.7 & 4 & 11.5 & 16 \\
10 & 11.4 & 9 & 11.7 & 7 & 10.8 & 15 \\
11 & 10.1 & 8 & 11.7 & 7 & 10.8 & 15 \\
12 & 11.4 & 9 & 10.0 & 6 & 3.6 & 5 \\
13 & 1.3 & 1 & 6.7 & 4 & 5.8 & 8 \\
14 & 10.1 & 8 & 0 & 0 & 8.6 & 12 \\
15 & 7.6 & 6 & 10.0 & 6 & 3.6 & 5 \\
16 & 5.1 & 4 & 1.7 & 1 & 2.2 & 3 \\
17 & 0 & 0 & 5.0 & 3 & 0 & 0 \\
18 & 0 & 0 & 0 & 0 & .7 & 1 \\
Total & 0 & 0 & 1.7 & 1 & $\mathbf{1 0 0 . 0}$ & $\mathbf{1 3 9}$ \\
\hline & $\mathbf{1 0 0 . 0}$ & $\mathbf{7 9}$ & $\mathbf{1 0 0 . 0}$ & $\mathbf{6 0}$ & & \\
\hline
\end{tabular}

Table 2: Distribution of the Sample by Gender

\begin{tabular}{|lcccccc|} 
& \multicolumn{2}{c}{ PRIVATE } & \multicolumn{2}{c|}{ PUBLIC } & \multicolumn{2}{c|}{ Total } \\
Female & $\%$ & $\mathbf{n}$ & $\%$ & $\mathbf{n}$ & $\%$ & $\mathbf{n}$ \\
Male & 28.1 & 39 & 26.6 & 36 & 54 & 75 \\
Total & 28.1 & 40 & 17.3 & 24 & 46 & 64 \\
& $\mathbf{5 6 . 9}$ & $\mathbf{7 9}$ & $\mathbf{4 3 . 1}$ & $\mathbf{6 0}$ & $\mathbf{1 0 0 . 0}$ & $\mathbf{1 3 9}$ \\
\hline
\end{tabular}

therefore, dichotomous, representing either continuous residence with the kinship caregiver or removal to a setting other than the biological parent. The independent variable was treatment, consisting of the categories privateand public. A retrospective cohort design was used to test the hypothesis that children from the privategroup would experience greater stability of placement than those from the public.

\section{Statistical Method}

The groups were compared on three variables: age, sex, and race. Ages were compared using a t-test. Chi-square analysis was used for both sex and race. To compare outcomes between groups, placement stability was cross-tabulated with treatment and the hypothesis was tested using the chi-square distribution. 


\begin{tabular}{|c|c|c|c|c|c|c|}
\hline \multirow{2}{*}{$\begin{array}{l}\text { Race } \\
\text { Black }\end{array}$} & \multicolumn{2}{|c|}{ PRIVATE } & \multicolumn{2}{|c|}{ PUBLIC } & \multicolumn{2}{|c|}{ Total } \\
\hline & 77.2 & 61 & 86.7 & 52 & 81.3 & 113 \\
\hline White & 20.0 & 16 & 11.7 & 7 & 16.5 & 23 \\
\hline Native American & 1.3 & 1 & 0 & 0 & .7 & 1 \\
\hline Multiracial & 1.3 & 1 & 0 & 0 & .7 & 1 \\
\hline Other & 0 & 0 & 1.3 & 1 & .7 & 1 \\
\hline Total & 100.0 & 79 & 100.0 & 60 & 100.0 & 139 \\
\hline
\end{tabular}

\section{RESULTS}

Analysis of the demographic variables showed little indication of difference between the groups. The mean age of participants in the private(8.63years) and the public groups (8.95 years) did not differ significantly and, perhaps more importantly, the squared point bi-serial correlation revealed that only . $2 \%$ of the variance in age could be explained by group membership $\left(t=.456, p=649, r_{P B I}=039\right)$. No significant relationship between group membership and gender was observed and the relationship between these two variables was also quite weak $\left(\chi^{2}=1.367, p=242\right.$, $\Phi=100)$. The groups also did not differ significantly by racial composition $\left(\chi^{2}=\right.$ 1.889, $p=169$, Cramer's V=118). The sample was thus similar to those from other studies where children were seven to eight years old, evenly distributed between the genders, and predominantly African-American.

On the outcome placement stability variable, 69 of the 78 private participants (88.5\%) remained in kinship care at the end of the study period. In comparison, 42 of the 54 public participants remained with relatives, a total of $77.8 \%$ (Table 4). Seven participants were excluded because of missing data. The results were significant at the .10 level $\left(\chi^{2}=2.723, p=10, \Phi=144\right)$.

\begin{tabular}{|c|c|c|c|c|}
\hline & \multicolumn{2}{|c|}{ PRIVATE } & \multicolumn{2}{|c|}{ PUBLIC } \\
\hline & $\mathrm{N}$ & $\%$ & $\mathrm{~N}$ & $\%$ \\
\hline No Disruption & 69 & 88.5 & 42 & 77.8 \\
\hline Disruption & 9 & 11.5 & 12 & 22.2 \\
\hline
\end{tabular}

The $p=10$ al pha level was selected over the traditional $p<05$ criterion to offset the probability of a Type II error. Stevens (1999, p. 135) suggests that alpha levels of .10 or even .15 may be appropriate in certain contexts. As Stevens points out, small and medium effect sizes are very common in social science research. Failure to reject null hypotheses based on stringent alpha levels may thus dissuade researchers from engaging in inquiry in new areas of research that may ultimately prove fertile (Stevens, 1999, p. 126). As such, relatively liberal al pha levels are often appropriate for studies that represent initial inquiries into new research problems. 
It is also instructive to consider the practical significance of this finding in the context of power versus risk reduction (Stevens, 1999, p. 135). Given that costs of the two programs under consideration in this study are similar, power should take precedence over the risk of falsely rejecting a null hypothesis. Rejecting a true null hypothesis would only lend support to a program of similar cost, which also produced a somewhat more positive outcome in the sample data. If, indeed, the more than $10 \%$ improvement is supported in future studies with larger samples, this could make an important difference for many children in state custody. In a group of 50,000 (not unrealistic to imagine in the future given today's escalating child welfare population), conditions for 5,000 would be improved. This suggests that proper program selection could have a substantial positive effect for thousands of children.

\section{DISCUSSION}

This study supports the efficacy of the private over the public program. This suggests that child welfare practitioners should be actively involved in developing and evaluating kinship programs. Research should focus on outcomes between models and the elements of those models. For example, it may be that the weekly support groups offered to caregivers in the private program were critical to their ability to cope with stress and make effective family management decisions. Alternatively, it is likely that the legal consultation and representation offered by the program helped participants make sound decisions while navigating the court system, enhancing the probability of placement stability.

The success of kinship programs does not mean that practitioners should expect placement with a relative to ensure success. The programs in this study provide intensive, specialized support. These supportive conditions often do not exist where specialized programs are not in place. Although practitioners should always consider kinship placements, they should not assume results such as those in this study.

Practitioners should develop initiatives using models and concepts that have been effective elsewhere. Outcomes should be evaluated and results disseminated. Advocacy efforts will need to include special funding allocations, policy accommodations, and some restructuring of service delivery systems.

Researchers should conduct evaluations using larger samples, more sophisticated designs, more informative statistical methods, and more comprehensive variables that use higher levels of measurement. Additional questions need to be answered regarding family assessment techniques, referral patterns, service utilization patterns, and service delivery systems.

Protocols for program comparison should be developed and used across studies. An example would be a table of program characteristics that includes a typology such as theone suggested by Scannapieco (1999) and developed in this article. The table could serve as a basis for program comparison. An example, Protocol for Model Comparison (PMC), is included in Table 5.

The PMC illustrates program differences in the current study. In the first dimension, formality/informality, both programs are formal. The programs offer different services (dimension 2). One consists of state workers (public) and the other of private 
employees (private) (dimension 3). Comparisons could not be made in the fourth dimension because one program lacked a clear statement of philosophy. Future studies could help to determine characteristics that are most crucial to outcome.

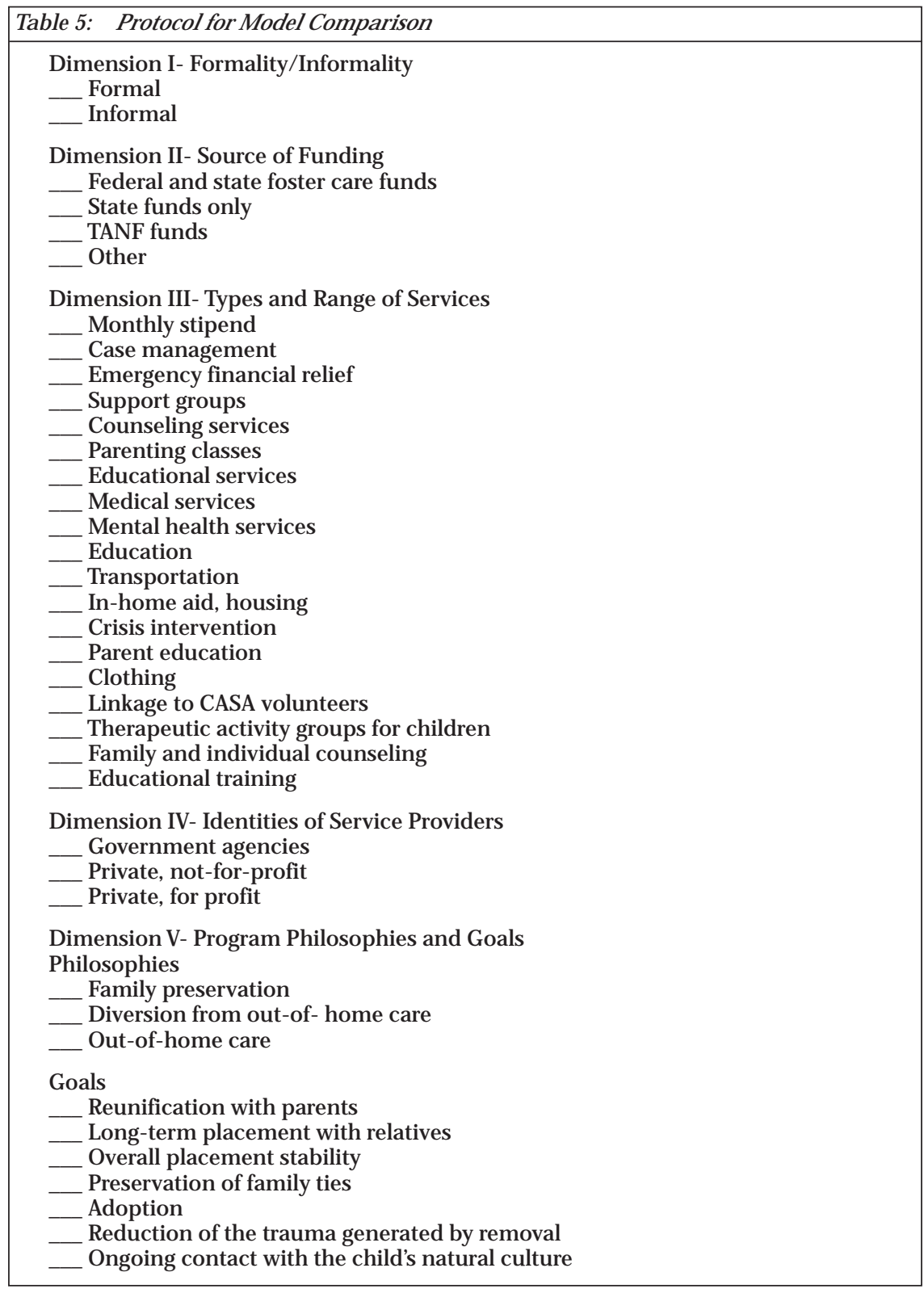




\section{LIMITATIONS}

The study was limited in a number of ways. It would have been desirable, for example, to include data from a traditional foster care group. Unfortunately, the structure of the record-keeping system between the juvenile court and the state child welfare department prevented the researchers from accessing foster care data.

Next, archival data limited the selection of variables and predetermined the level of measurement. This precluded the use of more complex statistical methods and restricted access to information that might have been available with higher levels of measurement.

Another limitation was the manner in which original data were collected. Study staff received extensive training to assure consistency in data collection. Various workers from different sources collected the original data, introducing the possibility of inter-rater reliability problems.

The outcome variable was also limited. The assumption that relocation of a child into a setting other than the care of a biological parent is detrimental may not be accurate. For example, a move to a more restrictive placement may be desirable when the services available are necessary to improve the child's condition. Furthermore, it cannot be assumed that return to the biological parents is always desirable.

A final limitation involved the referral process into the programs. Some children $(n=3)$ in the public program had originally been referred to the private, but either their families had elected not to participate, or the children were screened out because they failed to meet selection criteria. Criteria included a continuum of conditions, ranging from families showing no need for specialized intervention, to being in an extreme state of crisis. Depending on the number of children who did not participate in the private program for any of these reasons, outcomes might have been affected.

Despite the limitations of this study, it provides valuable information in an area characterized by its paucity, constituting a valuable foundation for future research. Different designs, such as experimental or single-subject, may provide additional insight into effectiveness. Future studies should use live data sources with levels of measurement chosen to provide optimal conditions. Researchers should use multiple outcome measures, including placement stability, psychosocial functioning, and family adjustment. Within the models of care, treatment integrity and patterns of service utilization should be examined. Differential effects should be considered among ethnic groups, genders, age groups, and groups of children with special needs.

\section{SUMMARY}

Kinship care is a promising, although partial, response to the growing pressure on the child welfare system. Placing children with relatives can help to minimize the trauma of removal, maintain supportive family relationships, and preserve consistency of cultural experience. It is a desirable alternative for children, families, and child welfare providers. 
There are various kinship care models, ranging from informal, unfunded arrangements to highly structured, well-funded interventions. Additional research is needed to identify effective models and their critical components. The current study supports the effectiveness of the private over the public program. These findings argue for continued research into program alternatives.

\section{References}

Ainsworth, M.S., Blehar, M.C., Waters, E., \&Wall, S. (1978). Patterns of attachment: A psychol ogi cal study of thestrangesituation. Potomac, MD: Lawrence Earlbaum.

Berrick, J.D. (1997). Assessing quality of care in kinship and foster family care. Family Relations: Interdisciplinary Journal of Applied Family Studies, 46(3), 273-280.

Berrick, J.D., Barth, R.P., \& Needell, B. (1994). A comparison of kinship foster homes and family foster homes: Implications for kinship foster care as family preservation. Children and Youth Services Review, 16(2), 33-64.

Bilaver, L.A., Jaudes, P. K., Koepke, D., \& Goerge, R.M. (1999). The health of children in foster care. Social Service Review, 73(3), 401-416.

Chernoff, R., Combs-Orme, T., Risley-Curtiss, C., \& Heisler, A. (1994). Assessing the health status of children entering foster care. Pediatrics, 93(4), 594.

Child Welfare League of America. (1994). Kinship care: A natural bridge Washington, D.C.: Author.

Courtney, M.E. (1996). Kinship foster careand children's welfare: The California experience. Focus, 17(3), 4248.

Dubowitz, H. (1994). Kinship care: Suggestions for future research. Child Welfare, 73, 553-562.

Dubowitz, H., Feigelman, S., Zuravin, S., Tepper, V. Davidaon, N., \& Lichenstein, R. (1992). The physical health of children in kinship care. American Journal of Diseases of Children, 146, 603-610.

Dubowitz, H., Tepper, V., Feigelman, S., Sawyer, R., \& Davidson, N. (1990). The physical and mental health and educational status of children placed with relatives: Final report. Report prepared for the Maryland Department of Human Services and the Baltimore City Department of Social Services, Baltimore, Maryland.

Fahlberg, V. (1979). Attachment and separation. Lansing, MI: Department of Social Services.

Gabel, G. (1992). Preliminary report on kinship foster family profile. New York: Human Resources Administration, Child Welfare Administration.

Gleeson, J.P. (1999). Kinship care as a child welfare service: Emerging policy issues and trends. In R.L. Hegar \& M. Scannapieco (Eds). Kinship foster care: Policy, practice, and research (pp. 28-53). New York: Oxford University Press.

Gleeson, J.P., \& Craig, L.C. (1994). Kinship care in child welfare: An analysis of states' policies. Children and Youth Services Review, 16, 7-31.

Goldstein, J., Freud, A., \& Solnit, A.J. (1973). Beyond the best interests of thechild. New York: Free Press.

Hegar, R. L. (1999). The cultural roots of kinship care. In R.L. Hegar \&M. Scannapieco (Eds). Kinship foster care: Policy, practice, and research (pp. 17-27). New York: Oxford University Press.

Henry, J. (1999). Permanency outcomes in legal guardianships of abused/neglected children. Families in Society: The Journal of Contemporary Human Services, 80(6), 561-568.

Iglehart, A.P. (1994). Kinship foster care: Placement, service, and outcome issues. Children and Youth Services Review, 16(2), 107-122.

Ingram, C. (1996). Kinship care: From last resort to first choice. Child Welfare, 75(5), 550-556.

National Adoption Information Clearinghouse. (1997). Keeping thefamily treintact through kinship care. [Online]. Available: http://www.calib.com/naic/pubs/f_kinshi.htm.

Ross, J.L. (1995, May 25). Services for young foster children. Washington, D.C.: U.S. General Accounting Office (GAO/HEHS-95-114). 
Scannapieco, M. (1999). Formal kinship care practice models. In R.L. Hegar \& M. Scannapieco (Eds). Kinship foster care: Policy, practice, and research (pp. 71-83). New York: Oxford University Press.

Scannapieco, M., \& Hegar, R.L. (1996). A non-traditional assessment framework for formal kinship homes. Child Welfare, 75(5), 567-579.

Scannapieco, M., Hegar, R.L., \& McAlpine, C. (1997). Kinship care and foster care: A comparison of characteristics and outcomes. Families in Society: The Journal of Contemporary Human Services, 78(5), 480488.

Scannapieco, M., \& Jackson, S. (1996). Kinship care: The African-American response to family preservation. Social Work, 41(2), 190-196.

Stevens, J. (1999). Intermediate statistics (2 $2^{\text {nd }}$ ed.). Mahwah, NJ: Lawrence Erlbaum Associates.

Task Force on Permanency Planning for Foster Children, Inc. (1990). Kinship foster care: Thedouble edged dilemma. Rochester, NY: Author.

Thornton, J.L. (1991). Permanency planning for children in kinship foster homes. Child Welfare 70(5), 593601.

United States Department of Health and Human Services, National Center on Child Abuse and Neglect. (1996). No place to call home: Discarded children in America. Washington, D.C.: U.S. Government Printing Office.

Verschueren, K., Marcoen, F., \& Schoefs, A. (1996). The internal working model of self, attachment, and competence in five-year-olds. Child Development, 67(5), 2493-2511.

Wilhelmus, M. (1998). Mediation in kinship care: Another step in the provision of culturally relevant child welfare services. Social Work, 43(2), 117-125.

Wilson, D.B. (1999). Kinship carein family-serving agencies. In R.L. Hegar \& M. Scannapieco (Eds). Kinship foster care: Policy, practice, and research (pp. 84-92). New York: Oxford University Press.

Wulczyn, F., \& Goerge, R.M. (1990). Public policy and theand thedynamics of foster care: A multi-sitestudy of placement histories. Washington, DC: U.S. Department of Health and Human Services, Office of Assistant Secretary for Planning and Evaluation.

\title{
Author's Note:
}

Address correspondence to: Rodney A. Ellis, Ph.D., The University of Tennessee, College of Social Work, 193E Polk Street, Nashville, TN 37210. E-mail: rellis5@utk.edu.

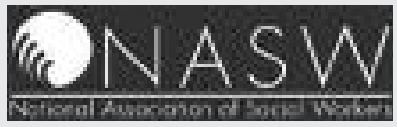

\section{National Association of Social Workers (NASW) Indiana Chapter}

\author{
Annual State Conference \\ October 9-11, 2002
} Marriott Hotel - Indianapolis, Indiana 